\title{
Measurements of the Sound Absorption Coefficient of Auditorium Seats for Various Geometries of the Samples
}

\author{
Jarosław RUBACHA, Adam PILCH, Marcin ZASTAWNIK \\ AGH University of Science and Technology \\ al. A. Mickiewicza 30, 30-059 Kraków, Poland; e-mail: jrubacha@agh.edu.pl \\ (received October 11, 2011; accepted October 4, 2012)
}

\begin{abstract}
This paper presents the results of measurements of the sound absorption coefficient of auditorium seats carried out in the laboratory using two methods. In the first one, small blocks of seats in various arrangements were studied in a reverberation chamber to determine the absorption coefficient of an auditorium of infinite dimensions. The results were compared to the values of the absorption coefficient measured using the second method, which involved samples enclosed within a frame screening the side surfaces of other auditorium blocks. The results of both methods allowed for the assessment of the sound absorption coefficient of an auditorium of any dimensions while taking into account the sound absorption by the side surfaces. The method developed by the authors will simplify the currently known measurement procedures.
\end{abstract}

Keywords: sound absorption, room acoustics, modelling of acoustic parameters.

\section{Introduction}

The goal of the research presented in this article was to determine the sound absorption coefficients of an auditorium, which are used in computer simulations of room acoustic parameters. The existing relationships used to determine the reverberation time, which are based on the statistical theory of acoustic fields, require the sound absorption coefficient as one of the basic parameters. Furthermore, programs used to simulate acoustic parameters of rooms, which are based on statistical models and the use of ray tracing and image source methods, require sound absorption coefficients as input parameters in the calculations. In concert halls and similar rooms, the auditorium constitutes an element with the greatest acoustic absorption and thus has the greatest effect on room reverberation conditions. This was confirmed by research conducted by KAMISIŃSKI (2010), who presented the result of simulation of acoustic parameters of concert halls for different sound absorption coefficients of the auditorium chairs. Therefore, to determine appropriate values of the sound absorption coefficient of an auditorium it is essential to increase the precision of prediction of acoustic parameters of a given interior.

Sound absorption by an auditorium has been studied among others by Beranek, Davies and Bradley, who revealed a number of problems that occurred during the measurements. The arrangements of seats in an auditorium is a spatial structure in that the large number of protruding edges increases the absorption of the entire auditorium as compared to the absorption capacity of a single seat. The research conducted by PILCH (2011) and KAMIsIŃsKI et al. (2012) on sound diffusers showed that sound absorption by spatial structures depends on their material properties and geometry, especially at low frequencies.

The existing method of measuring the sound absorption coefficient in a reverberation chamber in accordance with the standard (ISO, 2003) allows for a measurement to be performed on up to 24 seats, which represents a small proportion of the number of seats in concert halls ranging from several hundred to several thousand. Also, the seats in the auditoria are arranged in various patterns with different numbers of seats per the surface area and different auditorium profiles or arrangement of blocks.

The research conducted by BERANEK (1969) in different rooms showed that the sound absorption of an auditorium is proportional to its surface area. He also suggested that in order to calculate the absorption coefficient, the surface area of the block should be increased by a strip of $0.5 \mathrm{~m}$ around it in order to take into account the effect of sound absorption by the side surfaces. 
DAVIES et al. (1994) presented in their paper a method for determining the sound absorption coefficient of an auditorium with and without the use of screens covering the side surfaces of the sample, which made it possible to determine the effect of the side surfaces on the sound absorption by the auditorium. A similar measurement method is set forth in the standard (ISO, 2003), according to which the sound absorption coefficient is determined for a sample with reflecting screens. This method, however, does not take into account the effect of sound absorption by the side surfaces.

Note though that BRADLEY $(1992 ; 1996)$ showed in his papers that the sound absorption coefficient of an auditorium block is linearly dependent on the ratio of the sample perimeter to its surface area. In this method, the relationship (1) was used, which was earlier derived for flat samples.

$$
\alpha=\beta\left(\frac{P}{S}\right)+\alpha_{\infty}
$$

where $\alpha$ is the absorption coefficient of the auditorium block with perimeter $P$ and surface area $S, \beta$ is the regression coefficient, and $\alpha_{\infty}$ is the sound absorption coefficient of a surface of infinite dimensions.

\section{Description of the study}

The measurements of the sound absorption coefficient were conducted in a reverberation room. The reverberation times were determined by integrating the impulse response. The absorption coefficient was calculated from the formula given in the standard (ISO, 2003) for $1 / 3$ octave bands and then averaged to obtain values for $1 / 1$ octave bands.

The study of sound absorption using the method presented by Bradley (method 1) was carried out for three types of empty seats and for two types of occupied seats. Table 1 contains details of the design of the seats studied. For each type of seats, the measurements were performed for five arrangements of auditorium blocks with different $P / S$ values and a constant distance between the rows of $0.90 \mathrm{~m}$. The surface area used in the calculations was taken as a projection of the auditorium block on the floor. The sound absorption coefficients for each frequency band were used to determine the regression coefficients. Then, substituting the calculated values of the coefficient into formula (1), the dependence of the sound absorption coefficient of the auditorium on the size of the block was determined for each frequency band with a width of one octave.

The relationships discovered made it possible to extrapolate the absorption measurements for small blocks of seats to block dimensions of real halls and to areas of infinite dimensions. Figure 1 shows that for small $P / S$ values the sound absorption coefficient values are also small, which is due to the fact that in blocks with large surface areas the effect of sound absorption by side surfaces of the auditorium is smaller.

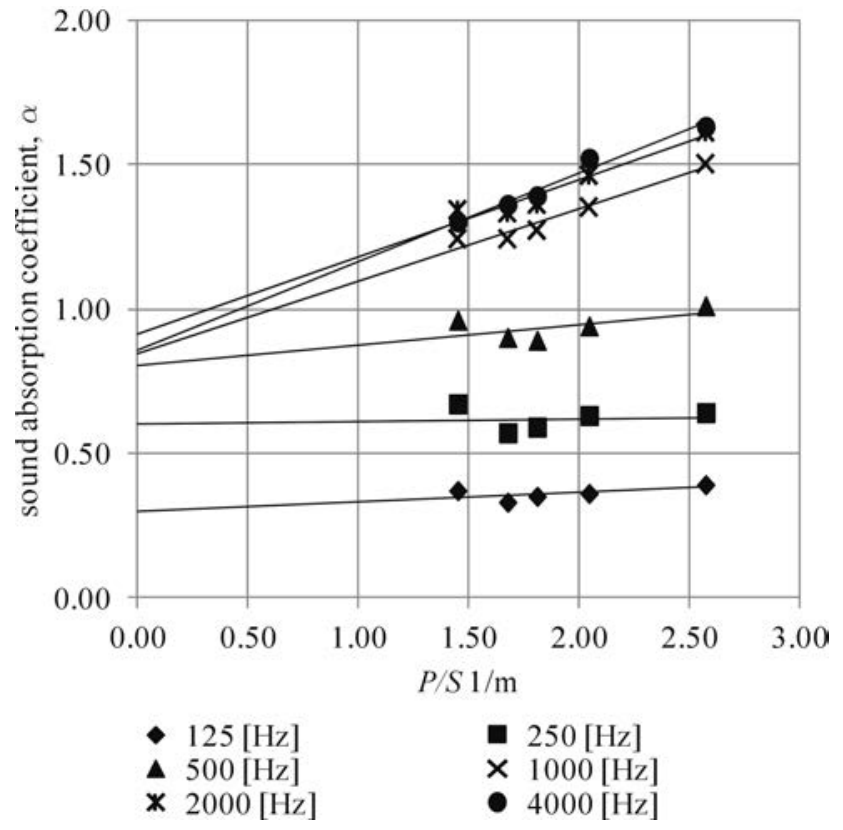

Fig. 1. Sound absorption coefficient $\alpha$ of seat 2 as a function of the perimeter to surface area ratio $P / S$.

Table 1. Design of the seats.

\begin{tabular}{|c|l|l|l|l|}
\hline No. & Chair element & \multicolumn{1}{|c|}{ Seat 1} & \multicolumn{1}{c|}{ Seat 2} & \multicolumn{1}{c|}{ Seat 3} \\
\hline 1 & Main structure & $\begin{array}{l}\text { Solid wood, } \\
15 \mathrm{~mm} \text { plywood }\end{array}$ & Solid wood & $\begin{array}{l}\text { Steel pipes, } \\
12 \mathrm{~mm} \text { profiled plywood }\end{array}$ \\
\hline 2 & Seat & $\begin{array}{l}120 \mathrm{~mm} \text { PU foam, } \\
\text { polyester fabric upholstery, } \\
10 \mathrm{~mm} \text { plywood at the bottom }\end{array}$ & $\begin{array}{l}50 \mathrm{~mm} \text { PU foam, } \\
\text { polyester fabric upholstery, } \\
\text { fabric at the bottom }\end{array}$ & $\begin{array}{l}15 \mathrm{~mm} \text { PU foam, } \\
\text { polyester fabric upholstery, } \\
12 \mathrm{~mm} \text { profiled plywood }\end{array}$ \\
\hline 3 & Back rest & $\begin{array}{l}50 \mathrm{~mm} \text { PU foam, } \\
\text { polyester fabric upholstery, } \\
10 \mathrm{~mm} \text { plywood at the back }\end{array}$ & $\begin{array}{l}30 \mathrm{~mm} \text { PU foam, } \\
\text { polyester fabric upholstery, } \\
\text { fabric at the back }\end{array}$ & $\begin{array}{l}15 \mathrm{~mm} \text { PU foam, } \\
\text { polyester fabric upholstery, } \\
12 \mathrm{~mm} \text { profiled plywood }\end{array}$ \\
\hline 4 & $\begin{array}{l}\text { Chair dimensions } \\
(\mathrm{W} \times \mathrm{L} \times \mathrm{H}) \mathrm{mm}\end{array}$ & $500 \times 570 \times 920$ & $510 \times 560 \times 840$ & $620 \times 570 \times 840$ \\
\hline
\end{tabular}


Figure 1 shows the dependence of the sound absorption coefficient on the $P / S$ ratio for seat 2. The study was conducted for $P / S$ values ranging from 1.30 to 2.60 , whereas the $P / S$ values of the average auditorium blocks in performance halls most often fall within a range from 0.30 to 1.00 .

The sound absorption coefficient was also studied using the second method in which the absorption coefficient was measured for samples mounted in a corner of the reverberation chamber (method 2). A reflecting frame with a height the same as that of the sample studied screened the sides of the sample. The frame was intended to reduce the effect of sound absorption by the side surfaces of the sample. The method of the sample mounting was similar to the assembly of type $\mathrm{J}$ as specified in the standard. For the calculation of the sound absorption coefficient, the same sample surface area was adopted as in the first method, i.e. the projection area of the block on the auditorium floor. The study of seats with spectators was performed for seats 1 and 2, whereas the study without spectators was performed on all three types of seats.

\section{Results}

The relationships determined by the first method were used to determine the sound absorption coefficients of the auditorium of an infinite surface area. The absorption coefficient results were then compared with those obtained by the method employing the reflecting frame. Figures 2 and 3 show the sound absorption coefficients with and without audience.

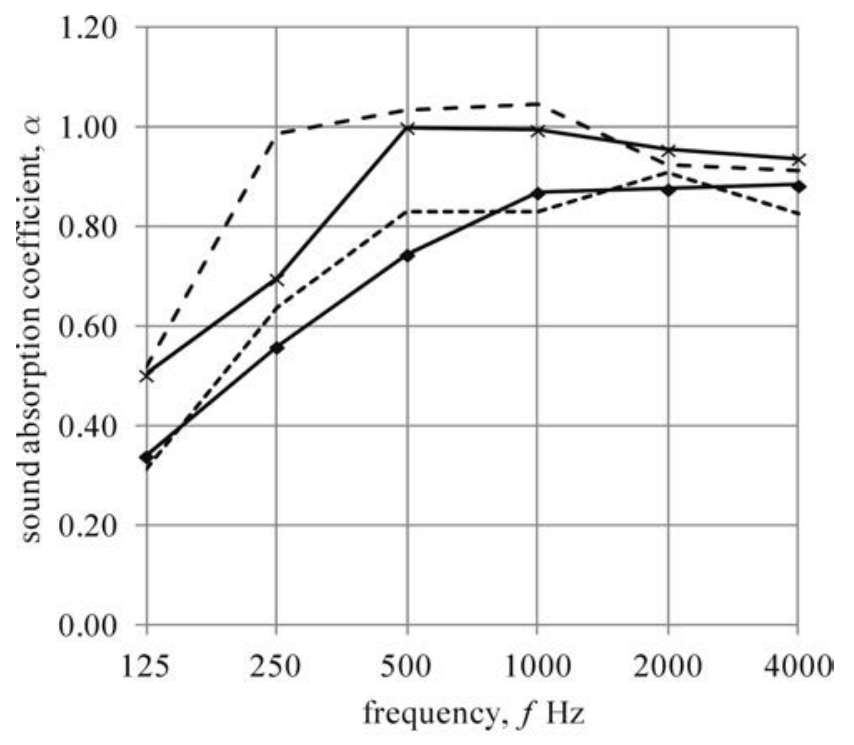

- - - Seat $1-$ method $1 \longrightarrow$ Seat $1-\operatorname{method} 2$

----Seat 2 - method $1 \longrightarrow$ Seat 2 - method 2

Fig. 2. Sound absorption coefficients determined by two methods for seats with audience.

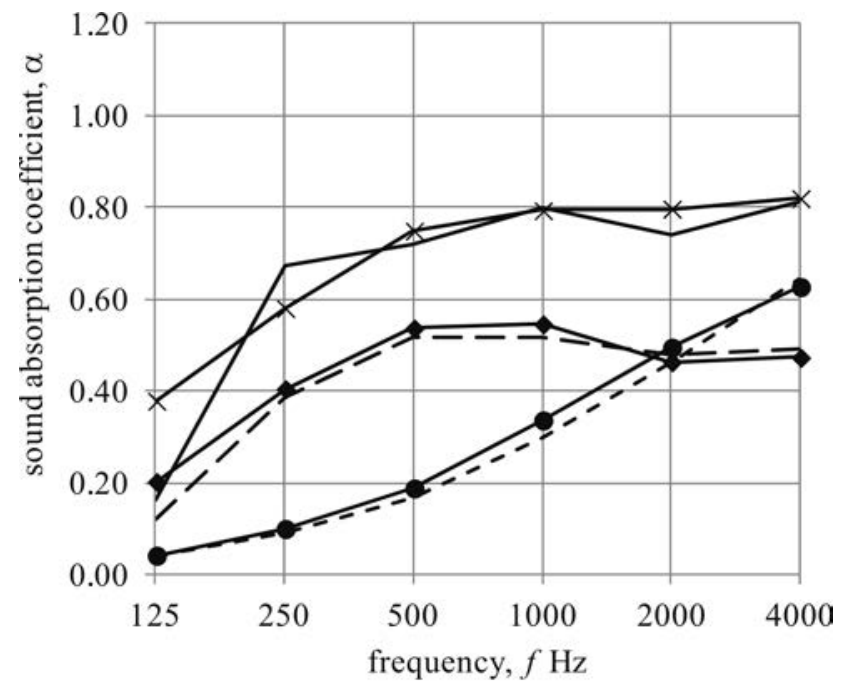

- Seat $1-$ method $1 \rightarrow$ Seat $1-\operatorname{method} 2$
-- Seat $2-\operatorname{method} 1 \rightarrow$ Seat $2-\operatorname{method} 2$
--- Seat $3-\operatorname{method} 1 \rightarrow-$ Seat $3-\operatorname{method} 2$

Fig. 3. Sound absorption coefficients determined by two methods for seats without audience.

As seen, the absorption coefficients determined using these methods have similar values. Hence, it can be concluded that both methods used in the study make it possible to eliminate the effect of sound absorption by the side surfaces and to determine the absorption coefficient of an auditorium sample of infinite dimensions.

In order to simplify the procedure and reduce the number of measurements, the absorption coefficient can be measured for two configurations of the sample. The measurement on the sample with a reflecting frame can be used to calculate the absorption coefficient of an auditorium of infinite dimensions $(P / S=0)$, while the measurement conducted for a sample of the same arrangement and dimensions, but without the reflecting frame around and positioned in the middle of the reverberation chamber, provide the sound absorption coefficient of an auditorium block of known dimensions and a given $P / S$ value. Then, for each frequency band, a linear Eq. (2) connecting the two points with known values of the absorption coefficient $\alpha$ and known $P / S$ values is determined, as shown in Fig. 4.

The resulting equations allow determining the sound absorption value $\alpha_{1}$ for auditorium blocks of any size according to Eq (2).

$$
\alpha_{1}=\beta_{1}\left(\frac{P}{S}\right)+\alpha_{\infty 1}
$$

To enable the use of the obtained sound absorption coefficients as input data for the model of the acoustic parameters of the room, the absorption coefficients 


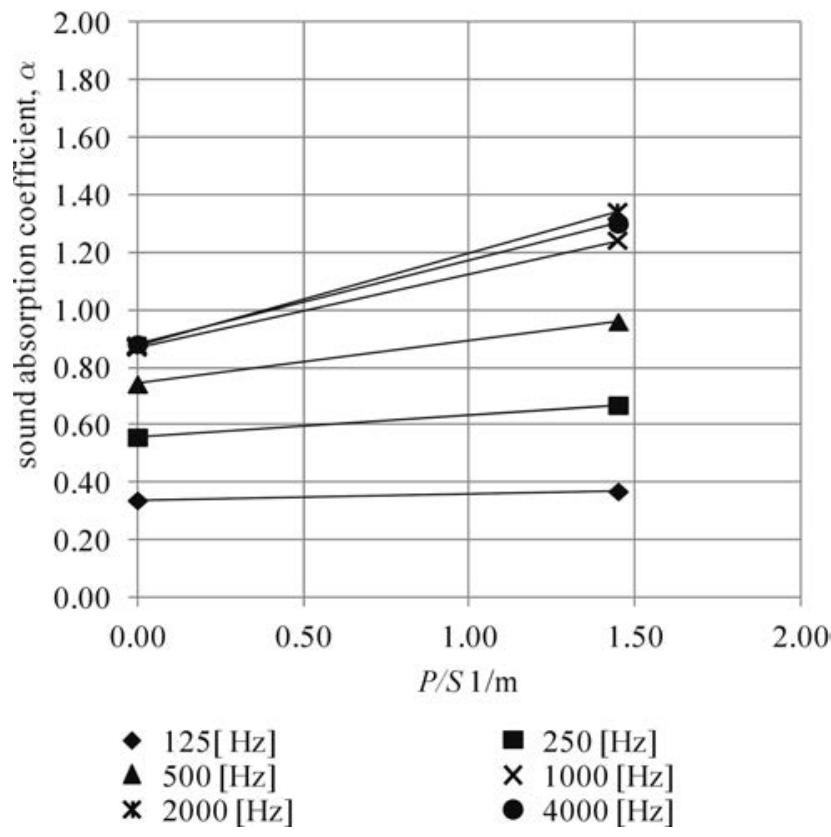

Fig. 4. The sound absorption coefficients and $P / S$ values used to determine the $\beta_{1}$ coefficients.

must be determined for the top and side surfaces of the auditorium sector as shown in Fig. 5.

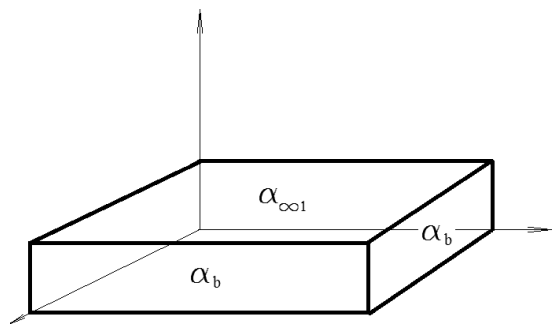

Fig. 5. Absorption coefficients of the top and side surfaces of a model of the auditorium block.

The results of the study show that the absorption coefficient of the top surface of the auditorium block has values similar to those of the sample of infinite dimensions, whereas for the side surfaces of the auditorium, the absorption coefficient can be determined using formula (3):

$$
S \alpha_{1}=S \alpha_{\infty 1}+S_{b} \alpha_{b},
$$

where $S$ is the surface area of the block projection on the floor, $S_{b}$ is the total side surface, $\alpha_{1}$ is the total sound absorption coefficient of the block, $\alpha_{\infty 1}$ is the absorption coefficient of the top surface and $\alpha_{b}$ is the sound absorption coefficient of the side surfaces. After transformation, the absorption coefficient of the side surfaces can be determined using the formula

$$
\alpha_{b}=\frac{\beta_{1}}{h},
$$

where $\beta_{1}$ is the slope of the line for a given octave and $h$ is the height of the auditorium block. In the case of an empty auditorium, the value of $h$ is taken to be equal to the height of the seats, whereas in the cases of an occupied auditorium, this value equals $1.2 \mathrm{~m}$, which corresponds to an average height of a seated person.

The graphs in Figs. 6 and 7 give the values of the sound absorption coefficient for the side surfaces of the tested seats with and without audience. They are the average absorption coefficients of all side surfaces, i.e. of the front, back, right and left ones. As seen, these values are lower than that for the top surface of the auditorium block. This is primarily due to the low absorption of the side surfaces of the auditorium blocks.

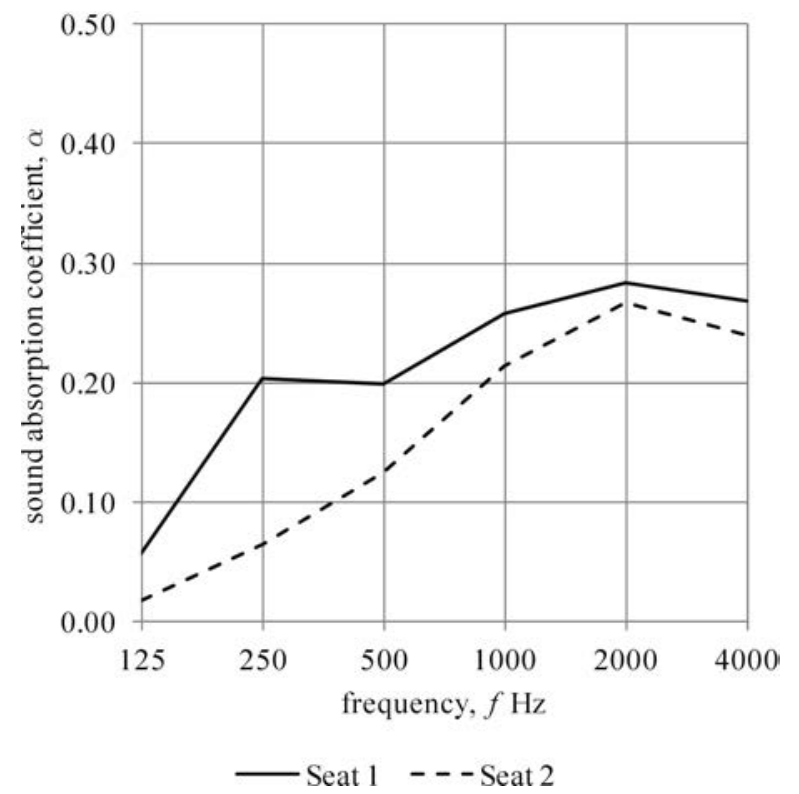

Fig. 6. The absorption coefficient values $\alpha_{b}$ for side surfaces of the auditorium block with an audience.

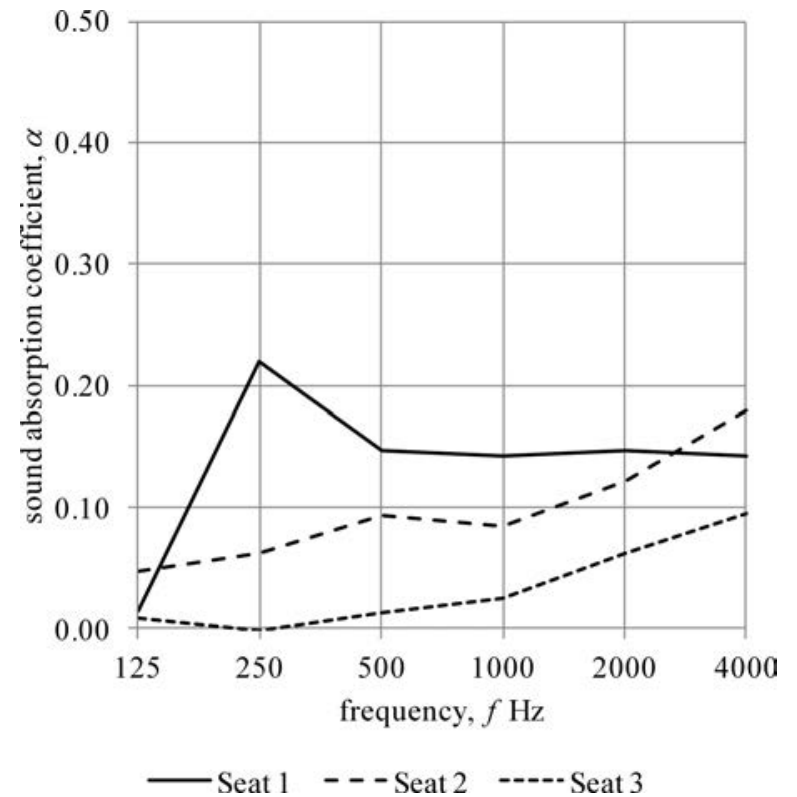

Fig. 7. The absorption coefficient values $\alpha_{b}$ for side surfaces of the auditorium block without audience. 


\section{Validation}

The method to determine the sound absorption coefficient of the auditorium block presented in this article was validated by comparing our values of the regression coefficients $\beta_{1}$ and the absorption coefficients $\alpha_{1}$ with the coefficients $\beta$ and $\alpha$ obtained by the Bradley's method (method 1).

The comparison (Fig. 8) showed that the greatest differences between the regression coefficients $\beta$ and $\beta_{1}$ occur in the $125-250 \mathrm{~Hz}$ frequency range where they exceed 0.15. For higher frequencies, the differences are smaller and do not exceed 0.10 . The coefficients $\alpha$ and $\alpha_{1}$ were also compared in order to assess the effect of differences in the regression coefficients on the sound absorption coefficients of the entire auditorium. The comparison was made for an auditorium block with a perimeter to area ratio $P / S$ ranging from 0.30 to 1.00 , which corresponds to the values most commonly encountered in performance halls. The sound absorption

a)

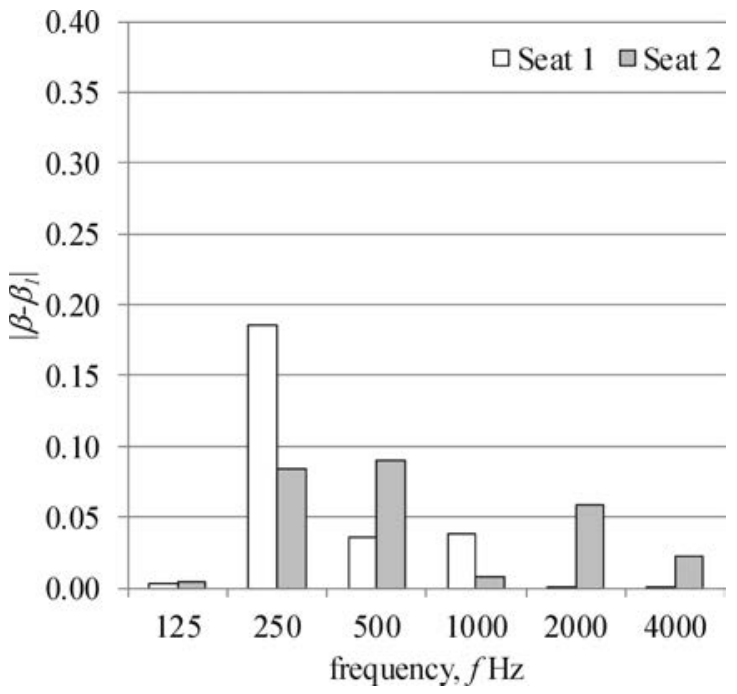

b)

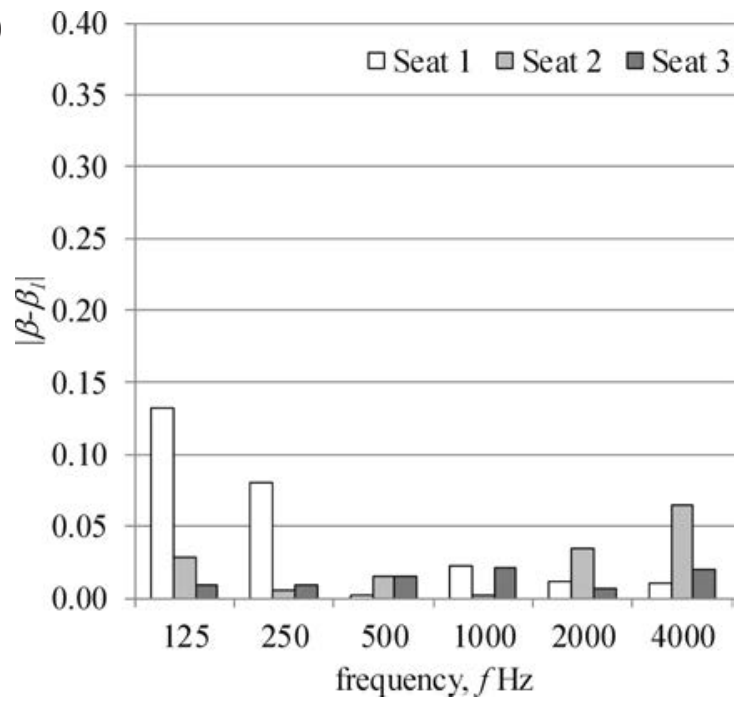

Fig. 8. Differences between the regression coefficients $\beta$ and $\beta_{1}$ for seats: a) occupied, b) unoccupied. coefficients were determined on the basis of Eqs. (1) and (2). To compare the results, the relative error $\delta_{\alpha}$ was calculated according to Eq. (5)

$$
\delta_{\alpha}=\left|\frac{\alpha-\alpha_{1}}{\alpha}\right| \cdot 100 \%
$$

where $\alpha$ is the sound absorption coefficient of the auditorium block obtained by the Bradley's method (method 1), while the absorption coefficient $\alpha_{1}$ was determined using method 2 . The results of the absorption coefficients are compared in Figs. 9 and 10.

a)

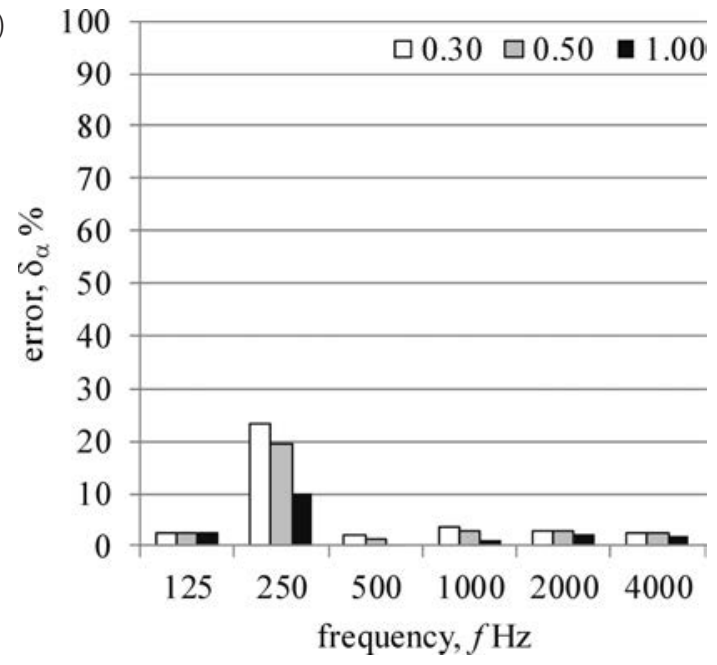

b)

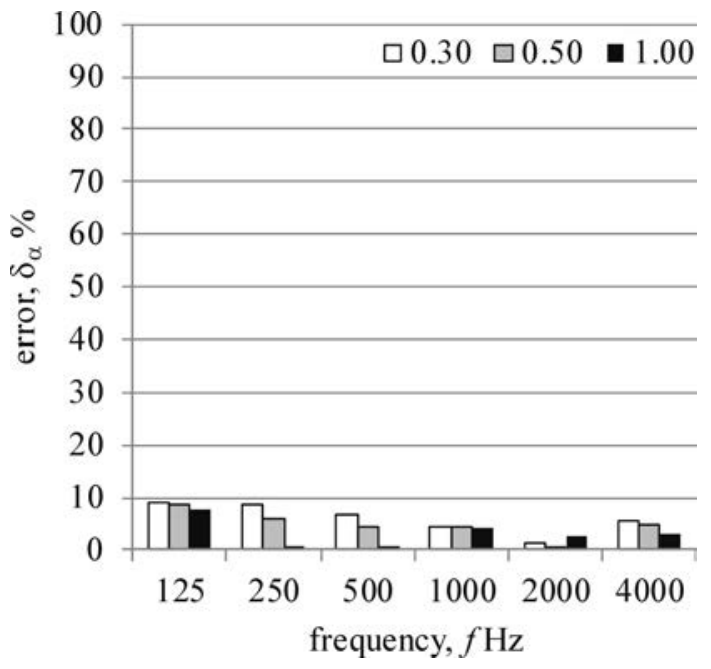

Fig. 9. Relative error for occupied seats at different values of $P(S:$ a) seat $1, \mathrm{~b})$ seat 2 .

The comparison of the sound absorption coefficients determined by the two methods showed that at frequencies above $250 \mathrm{~Hz}$ the differences do not exceed $10 \%$ for the occupied or the unoccupied seats. The greatest differences were noted for unoccupied seats at low frequencies, $125 \mathrm{~Hz}$ and $250 \mathrm{~Hz}$, and small $P / S$ values, where the error $\delta_{\alpha}$ is over $80 \%$. For occupied seats the largest discrepancies do not exceed $25 \%$.

The differences between the regression coefficients $\beta$ and $\beta_{1}$ have a direct effect on the sound absorp- 
a)

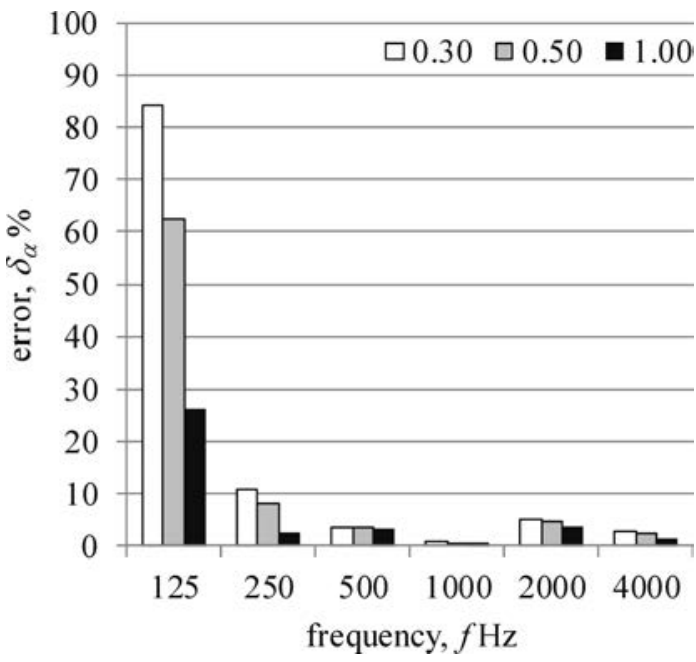

b)

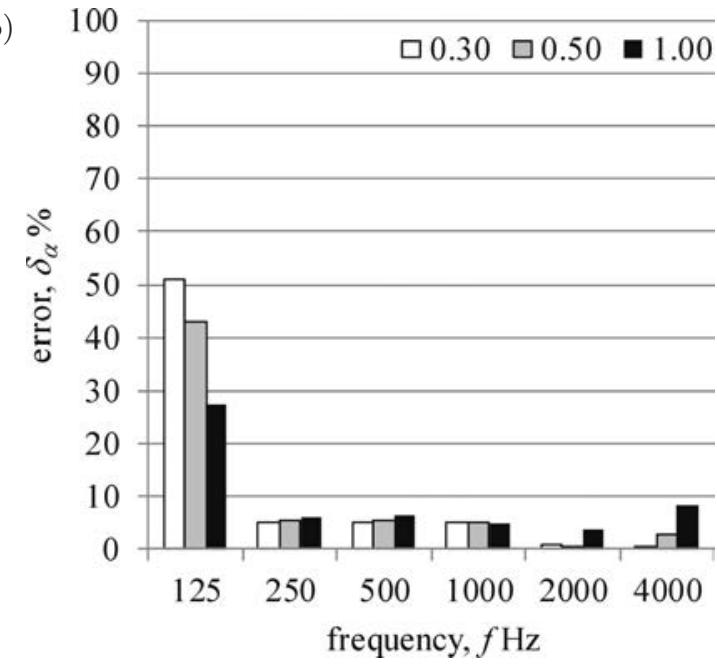

c)

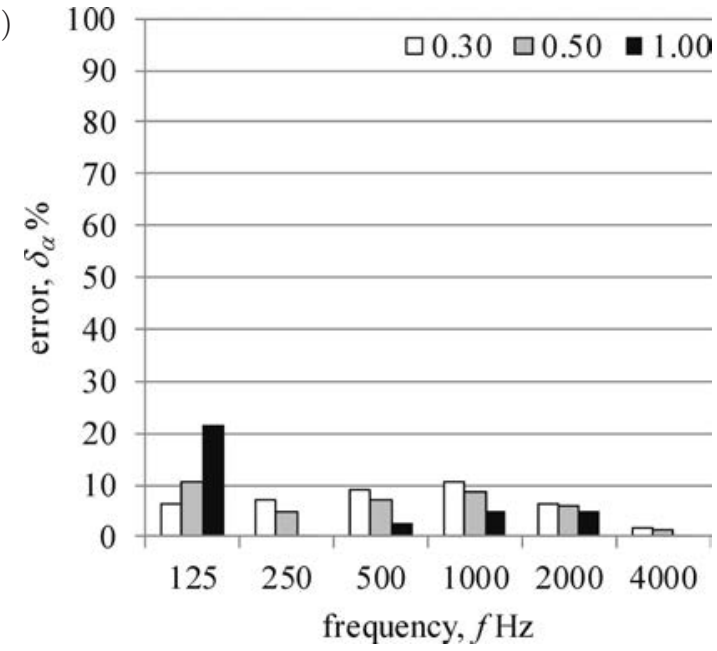

Fig. 10. Relative error for unoccupied seats at different values of $P(S$ : a) seat $1, \mathrm{~b})$ seat $2, \mathrm{c}$ ) seat 3 .

tion coefficients of the side surfaces $\alpha_{b}$ according to Eq. (4), which in turn translates into differences in the absorption coefficients of the entire auditorium block, especially at low frequencies.

Too small sample sizes and a non-uniform sound field in this frequency range may cause the differences.
This problem occurs in all methods of measurement carried out in reverberation rooms.

\section{Summary}

The results of the sound absorption coefficient of seats determined by two methods presented in this paper showed that both methods yield similar results at frequencies above $250 \mathrm{~Hz}$. This made it possible to propose a method to determine the absorption coefficient of an auditorium block of any dimensions based on two measurements of the sound absorption coefficient. It allows also to determine the sound absorption coefficients of the side surfaces of the block, which makes them applicable to acoustic models of rooms. The methods used to this point do not take into account the sound absorption by the side surfaces of the auditorium, as they do, for instance, in the method presented in the standard; or, as the Bradley's method, they require at least five measurements of the sound absorption coefficient. The method presented in this paper is similar to that used by Davies, which requires three measurements of the absorption coefficient and employs different values of the sample surface area in calculations.

\section{Acknowledgment}

This study was supported by Dean's grant No. 15.11.130.213 "The acoustic aspects of structures of audience in concert halls".

\section{References}

1. Beranek L.L. (1969), Audience and chair absorption in large halls. II, J. Acoust. Soc. Am., 45, 1, 13-19.

2. Bradley J.S. (1992), Predicting theater chair absorption from reverberation chamber measurements, J. Acoust. Soc. Am., 91, 3, 1514-1524.

3. Bradley J.S. (1996), The sound absorption of occupied auditorium seating, J. Acoust. Soc. Am., 99, 2, 990-995.

4. Davies W.J., Orlowski R.J., Lam Y.W. (1994), Measuring auditorium seat absorption, J. Acoust. Soc. Am., 96, 2, 879-888.

5. Kamisiński T. (2010), Acoustic Simulation and Experimental Studies of Theatres and Concert Halls, Acta Physica Polonica A, 118, 1, 78-82.

6. Kamisiński T., Brawata K., Pilch A., Rubacha J., Zastawnik M (2012), Sound Diffusers with Fabric Covering, Archives of Acoustics, 37, 3, 317-322.

7. Pilch A., Kamisiński T. (2011), The Effect of Geometrical and Material Modification of Sound Diffusers on Their Acoustic Parameters, Archives of Acoustics, 36, 4, 955-966.

8. ISO 354:2003 Acoustics - Measurement of sound absorption in a reverberation room. 